\title{
Evaluation of the Equality of Non-Polar Capillary Columns in GC/MS Analysis of Food Contact Plastics
}

\author{
Motoh Mutsuga*, Miku Yamaguchi, Yutaka Abe, Hiroshi Akiyama \\ Division of Food Additives, National Institute of Health Sciences, Tokyo, Japan \\ Email: *mutsuga@nihs.go.jp
}

Received June 25, 2013; revised July 25, 2013; accepted August 27, 2013

Copyright (C) 2013 Motoh Mutsuga et al. This is an open access article distributed under the Creative Commons Attribution License, which permits unrestricted use, distribution, and reproduction in any medium, provided the original work is properly cited.

\begin{abstract}
Non-polar capillary columns for GC/MS are widely utilized in the analysis of additives for food contact materials. Though various kinds of non-polar capillary columns are commercially available, the equality of their performance has not been verified. Herein, ninety-six additives for food contact plastics were analyzed using fifteen kinds of columns, and the peak separation, retention times, and peak areas of each additive were compared. The additives, with various chemical properties, comprised forty four plasticizers, twenty lubricants, twenty antioxidants, nine ultraviolet absorbers, and three other compounds. $10 \mu \mathrm{g} \cdot \mathrm{mL}^{-1}$ test solutions were prepared in acetone, and injected to the GC/MS. The fifteen columns were classified into five categories based on the chromatogram pattern and peak separation. To facilitate comparison of the retention time and detection sensitivity of the columns for the additives, the relative retention time (RRT) and relative peak area (RPA) were calculated by using dibutylphthalate or 4-tert-butylphenylsalicylate as an internal standard. The RRTs of the additives on each column were essentially similar. However, the RRT of the additives which were detected in the later stages differed slightly. Although the RPA of the plasticizers and lubricants were roughly similar, column-to-column differences were observed for certain additives, such as antioxidants and ultraviolet absorbers. Furthermore, certain fatty acids, antioxidants, two plasticizers, and two benzophenone type ultraviolet absorbers were not detected in the chromatograms of two columns.
\end{abstract}

Keywords: Non-Polar Capillary Column; GC/MS Analysis; Additives for Food Contact Plastics; Relative Retention Time; Relative Peak Area

\section{Introduction}

Gas chromatography/mass spectroscopy (GC/MS) is used world-wide as an analytical method for screening, identification, and quantification of various chemicals. GC/MS analysis is particularly suitable for identification and quantification of chemicals used for food contact materials. Quantification of the residual or migration levels of such chemicals is important for safety assurance and specification testing for national regulatory purposes. Recently, the quantification of plasticizers, antioxidants, alkyl phenols, and benzophenones has been reported using certain simultaneous analytical methods employing a non-polar capillary column [1-5]. In these reports, various non-polar columns such as HP-5MS, DB5-MS, and TRB-5MS were used based on the author's selection. The most commonly used non-polar capillary column is the $5 \%$ diphenyl polysiloxane/dimethyl polysiloxane phase type. However, various non-polar columns made

${ }^{*}$ Corresponding author. from several materials, such as the phenyl-arylene/imethyl polysiloxane phase and siloxane-carborane phase type are commercially available and used in GC/MS analyses. Furthermore, certain columns have also been developed for high temperature analysis. The characteristics of the columns vary widely depending on the technique of the manufacturers. Column catalogues provide comparisons of the performance of competitors. However, to date, these performance comparisons have not been verified.

Herein, ninety six additives used for food contact plastics were selected from the analytes studied in our previous reports [6-10] or from the positive list of EU [11], and a mixture of these chemicals was analyzed via GC/MS. The peak separation, the retention time, and the peak area of each additive were analyzed. Based on comparison of the performance parameters of fifteen columns, the equality of commercially available non-polar columns is verified herein. The properties of these columns are compared based on analysis of additives used for 
food contact plastics.

\section{Materials and Methods}

\subsection{Column}

Fifteen columns $(15 \mathrm{~m} \times 0.25 \mathrm{~mm}$, thickness $0.1 \mu \mathrm{m})$ were used in this study. HP-5MS, DB-5, DB-5HT, DB5MS, CP-Sil 8CB-MS (CP-Sil), and VF-5ms were purchased from Agilent Technologies (CA, USA). ZB-5 and ZB-5MS were purchased from Phenomenex (CA, USA). Rxi-5HT and Rxi-5Sil MS (Rxi-5) were purchased from Restek (PA, USA). Three lots (SL100102, SL110302, and SL110601) of Inert Cap 5MS/Sil (Inert Cap) were purchased from GL Sciences (Tokyo, Japan). TR-5MS was purchased from Thermo Fisher Scientific (PA, USA). BPX5 and HT5 were purchased from SGE Analytical Science (Victoria, Australia). 007-5MS was purchased from Quadrex (CT, USA). The detailed properties of these columns, such as the phase type, serial number, and accurate length are listed in Table $\mathbf{1 .}$

\subsection{Standard}

Ninety six standards were purchased from Tokyo Chemical Industry Co. Ltd., (Tokyo, Japan), Wako Pure Chemical Industries, Ltd., (Osaka, Japan), Kanto Chemical

Table 1 Properties of columns used in this study.

\begin{tabular}{|c|c|c|c|}
\hline Column & Phase type ${ }^{*}$ & Serial & Accurate length $^{* *}(\mathrm{~m})$ \\
\hline HP-5MS & (a) & US9609347H & 15.1 \\
\hline DB-5 & (a) & USB118311A & 16.5 \\
\hline ZB-5 & (a) & 191475 & 15.2 \\
\hline DB-5HT & (a) & USB330333A & 15.9 \\
\hline ZB-5HT & (a) & 220081 & 15.8 \\
\hline Rxi-5HT & (a) & 1041920 & 15.7 \\
\hline DB-5MS & (b) & US8649613A & 16.5 \\
\hline Rxi-5 & (b) & 1031442 & 15.0 \\
\hline \multirow[t]{3}{*}{ Inart Cap } & (b) & SL100102 & 15.7 \\
\hline & & SL110302 & 15.7 \\
\hline & & SL110601 & 15.7 \\
\hline CP-Sil & (b) & 2217061 & 15.0 \\
\hline VF-5ms & (b) & 2203501 & 15.2 \\
\hline TR-5MS & (b) & 12806A33 & 15.7 \\
\hline BPX5 & (b) & $12843 \mathrm{~A} 69$ & 15.5 \\
\hline 007-5MS & (b) & $110506 \mathrm{~F}$ & 16.1 \\
\hline HT5 & (c) & 12666B15 & 15.1 \\
\hline
\end{tabular}

*: (a) $5 \%$ diphenyl polysiloxane/dimethyl polysiloxane, (b) phenyl-arylene/dimethyl polysiloxane, (c) siloxane-carborane. ${ }^{* *}$ : Accurate length $=$ $($ diameter $\times 3.14 \times$ number of rolls $)+$ margin length.
Co. Inc. Co. (Tokyo, Japan), and Sigma Aldrich, Japan (Tokyo, Japan), comprising forty-four plasticizers, twenty lubricants, twenty antioxidants, nine ultraviolet absorbers, and three other compounds. These standards were chosen to be representative of typical additives for food contact plastics. Detailed specifications of the additives are listed in Tables 2 and $\mathbf{3 .}$

\subsection{Preparation of Test Solution}

Each stock solution was prepared by dissolving $20.0 \mathrm{mg}$ of standard in $20 \mathrm{~mL}$ of acetone or dichloromethane (1 $\left.\mathrm{mg} \cdot \mathrm{mL}^{-1}\right)$. Test solution A was prepared by mixing $1 \mathrm{~mL}$ of each stock solution of the plasticizers and three others, followed by dilution to $100 \mathrm{~mL}$ with acetone $(10$ $\left.\mu \mathrm{g} \cdot \mathrm{mL}^{-1}\right)$. Test Solution B was prepared by mixing $1 \mathrm{~mL}$ of each stock solution of lubricants and antioxidants and ultraviolet absorbers, followed by dilution to $100 \mathrm{~mL}$ with acetone $\left(10 \mu \mathrm{g} \cdot \mathrm{mL}^{-1}\right)$.

\subsection{GC/MS Conditions}

GC/MS analysis was carried out on an Agilent Technologies $7890 \mathrm{GC}$ coupled to a $5975 \mathrm{C}$ mass selective detector (MSD). Injections $(1 \mu \mathrm{L})$ were made in splitless mode via a 7693 autosampler. The column temperature was programmed to increase in increments of $20^{\circ} \mathrm{C} \mathrm{min}^{-1}$ from $50^{\circ} \mathrm{C}$ to $320^{\circ} \mathrm{C}$, at which point the temperature was held for $20 \mathrm{~min}$. The $\mathrm{GC}$ injection temperature was $250^{\circ} \mathrm{C}$ and the transfer line temperature was $280^{\circ} \mathrm{C}$. Helium was used as the carrier gas at a flow rate of $1.0 \mathrm{~mL}$ $\min ^{-1}$. The MSD was operated in scan mode, scanning from $\mathrm{m} / \mathrm{z} 40$ to 800 using electron impact ionization at 70 $\mathrm{eV}$. Tuning was performed in DFTPP tune mode.

\subsection{Measurement and Data Analyses}

Test solutions were injected three times each per column. The peak separation and column classification were based on comparison of the total ion chromatogram patterns. Retention times and peak areas of quantitative ions were obtained from each chromatogram. When an additive was detected as multi-peaks, the retention time of the highest peak was used for analysis. The relative retention time (RRT) and the relative peak area (RPA) were calculated by using dibutylphthalate (A17) in test solution A or 4-tert-butylphenylsalicylate (B47) in test Solution B as an internal standard.

\section{Results and Discussion}

\subsection{Peak Isolation and Column Classification}

Typical total ion chromatograms (TIC) of the test solution, obtained using the columns, are shown in Figures 1 and 2. Though generally similar, the chromatogram patterns exhibited subtle differences. Thus, based on 
Table 2. Properties of additives for test Solution A.

\begin{tabular}{|c|c|c|c|c|c|c|}
\hline Application & Type & No. & Additives & CAS No. & Molecular weight & Quantitative ion $(\mathrm{m} / \mathrm{z})$ \\
\hline \multirow[t]{44}{*}{ Plasticizer } & Adipate & $\mathrm{A} 1$ & Diisopropyladipate & $6938-94-9$ & 230 & 129 \\
\hline & & $\mathrm{A} 2$ & Dipropyladipate & $106-19-4$ & 230 & 171 \\
\hline & & $\mathrm{A} 3$ & Diisobutyladipate & $141-04-8$ & 258 & 129 \\
\hline & & A4 & Dibutyladipate & $105-99-7$ & 258 & 185 \\
\hline & & A5 & Dialkyladipate $^{*}$ & - & - & 129 \\
\hline & & A6 & Di(2-ethylhexyl)adipate & $103-23-1$ & 370 & 129 \\
\hline & & A7 & Heptylnonyladipate $^{*}$ & $68515-75-3$ & 370 & 129 \\
\hline & & A8 & Dioctyladipate & $123-79-5$ & 370 & 241 \\
\hline & & A9 & Dibenzyladipate & $2451-84-5$ & 326 & 91 \\
\hline & & A10 & Diisononyladipate $^{*}$ & $33703-08-1$ & 398 & 129 \\
\hline & & A11 & Diisodecyladipate $^{*}$ & $27178-16-1$ & 426 & 129 \\
\hline & Phthalate & A12 & Dimethylphthalate & $131-11-3$ & 194 & 163 \\
\hline & & A13 & Diethylphthalate & $84-66-2$ & 222 & 149 \\
\hline & & A14 & Diisopropylphthalate & $605-45-8$ & 250 & 149 \\
\hline & & A15 & Dipropylphthalate & $131-16-8$ & 250 & 149 \\
\hline & & A16 & Diisobutyl phthalate & $84-69-5$ & 278 & 149 \\
\hline & & A17 & Dibutylphthalate & $84-74-2$ & 278 & 149 \\
\hline & & A18 & Benzylbutylphthalate & $85-68-7$ & 312 & 149 \\
\hline & & A19 & Diisoheptylphthalate $^{*}$ & 41451-28-9 & 362 & 149 \\
\hline & & A 20 & Di(2-ethylhexyl) 1,2,3,6-tetrahydrophthalate & $2915-49-3$ & 394 & 124 \\
\hline & & A21 & Dicyclohexylphthalate & $84-61-7$ & 330 & 149 \\
\hline & & A22 & Di(2-ethylhexyl)phthalate & $117-81-7$ & 390 & 149 \\
\hline & & A23 & Diisooctylphthalate $^{*}$ & 27554-26-3 & 390 & 149 \\
\hline & & A24 & Dinonylphthalate $^{*}$ & $84-76-4$ & 418 & 149 \\
\hline & & A25 & Di(2-ethylhexyl)isophthalate & $137-89-3$ & 390 & 112 \\
\hline & & A26 & Di-n-octylphthalate & $117-84-0$ & 390 & 149 \\
\hline & & A27 & Di(2-ethylhexyl)terephthalate & $6422-86-2$ & 390 & 112 \\
\hline & & A28 & Diisononyl 1,2-cyclohexanedicarboxylate & $166412-73-8$ & 424 & 155 \\
\hline & & A29 & Diisononylphthalate & $28553-12-0$ & 418 & 149 \\
\hline & & $\mathrm{A} 30$ & Diisodecylphthalate $^{*}$ & $26761-40-0$ & 446 & 149 \\
\hline & & A 31 & Tri(2-ethylhexyl)trimellitate & $3319-31-1$ & 546 & 305 \\
\hline & Citrate & A32 & Tributylcitrate & 77-94-1 & 360 & 185 \\
\hline & & A33 & Acetyltributylcitrate & $77-90-7$ & 402 & 185 \\
\hline & Sebacate & A34 & Dibutylsebacate & $109-43-3$ & 314 & 241 \\
\hline & & A35 & Di(2-ethylhexyl)sebacate & $122-62-3$ & 426 & 185 \\
\hline & Glycol & A36 & 2,2,4-Trimethyl-1,3-pentanediol diisobutyrate & $6846-50-0$ & 286 & 71 \\
\hline & & A37 & Glyceroldiacetatelaurate & $30899-62-8$ & 316 & 159 \\
\hline & & A38 & Neopentylglycoldibenzoate & $4196-89-8$ & 312 & 105 \\
\hline & & A39 & Triethyleneglycoldi(2-ethylhexanoate) & $94-28-0$ & 402 & 171 \\
\hline & Other & $\mathrm{A} 40$ & Dibutylmaleate & $105-76-0$ & 228 & 99 \\
\hline & & A41 & Di(2-ethylhexyl)azelate & $103-24-2$ & 412 & 171 \\
\hline & & A42 & p-tert-Octylphenol & $140-66-9$ & 206 & 135 \\
\hline & & A43 & 2-Ethylhexyldiphenylphosphate & $1241-94-7$ & 362 & 251 \\
\hline & & A44 & Cresyldiphenylphosphate ${ }^{*}$ & $26444-49-5$ & 340 & 340 \\
\hline \multirow[t]{3}{*}{ Other } & & A45 & $n$-Tetradecanol & $112-72-1$ & 214 & 55 \\
\hline & & A46 & Nonylphenol & $25154-52-3$ & 220 & 107 \\
\hline & & A47 & Bisphenol A & $80-05-7$ & 228 & 213 \\
\hline
\end{tabular}

\footnotetext{
*: Mixture of two or more compounds.
} 
Table 3. Properties of additives for test Solution B.

\begin{tabular}{|c|c|c|c|c|c|c|}
\hline Application & Type & No. & Additives & CAS No. & Molecular weight & Quantitative ion $(\mathrm{m} / \mathrm{z})$ \\
\hline \multirow[t]{20}{*}{ Lubricant } & Amide & B1 & Hexadecanamide & $629-54-9$ & 255 & 59 \\
\hline & & $\mathrm{B} 2$ & 9-Octadecenamide & $301-02-0$ & 282 & 59 \\
\hline & & B3 & Octadecanamide & $124-26-5$ & 284 & 59 \\
\hline & & B4 & cis-13-Docosenamide & $112-84-5$ & 337 & 59 \\
\hline & & B5 & Docosanamide & $3061-75-4$ & 339 & 59 \\
\hline & Fatty ester & B6 & Ethyl- $n$-hexadecanoate & $628-97-7$ & 284 & 88 \\
\hline & & B7 & Ethyl $(Z, Z)-9,12$-Octadecadienoate & $544-35-4$ & 308 & 67 \\
\hline & & B8 & Ethyl-cis-9-octadecenoate & $111-62-6$ & 310 & 55 \\
\hline & & B9 & Ethyloctadecanoate & $111-61-5$ & 312 & 88 \\
\hline & Fatty acic & $\mathrm{B} 10$ & Dodecanoic acid & $143-07-7$ & 200 & 60 \\
\hline & & B11 & Tetradecanoic acid & $544-63-8$ & 228 & 60 \\
\hline & & B12 & Hexadecanoic acid & $57-10-3$ & 256 & 60 \\
\hline & & B13 & Octadecanoic acid & $57-11-4$ & 284 & 60 \\
\hline & Hydrocarbon & B14 & $n$-Tetradecane & $629-59-4$ & 198 & 71 \\
\hline & & $\mathrm{B} 15$ & $n$-Hexadecane & $629-73-2$ & 226 & 71 \\
\hline & & B16 & n-Octadecane & $593-45-3$ & 254 & 71 \\
\hline & & B17 & $n$-Eicosane & $112-95-8$ & 282 & 71 \\
\hline & & B18 & $n$-Docosane & $629-97-0$ & 310 & 71 \\
\hline & & B19 & $n$-Tetracosane & $646-31-1$ & 338 & 71 \\
\hline & & B20 & $n$-Hexacosane & $630-01-3$ & 366 & 71 \\
\hline \multirow[t]{20}{*}{ Antioxidant } & Phenol & B21 & 3-tert-Butyl-4-hydroxyanisole & $121-00-6$ & 180 & 165 \\
\hline & & B22 & 2,6-Di-tert-butyl-4-methylphenol & $128-37-0$ & 220 & 205 \\
\hline & & B23 & 2,6-Di-tert-butyl-4-ethylphenol & $4130-42-1$ & 234 & 219 \\
\hline & & B24 & 2,6-Di-tert-butyl-4-hydroxymethylphenol & $88-26-6$ & 236 & 221 \\
\hline & & B25 & 2,2'-Methylenebis(6-tert-butyl-4-methylphenol) & $119-47-1$ & 341 & 177 \\
\hline & & B26 & 2,2'-Ethylidenebis(4,6-di-tert-butylphenol) & $35958-30-6$ & 439 & 233 \\
\hline & & B27 & 2,2'-Methylenebis(4-ethyl-6-tert-butylphenol) & $88-24-4$ & 369 & 191 \\
\hline & & B28 & 4,4'-Methylenebis(2,6-di-tert-butylphenol) & $118-82-1$ & 425 & 409 \\
\hline & & B29 & 2,2'-Methylenebis[6-(1-mehtylcyclohexyl)-p-cresol] & $77-62-3$ & 420 & 420 \\
\hline & & $\mathrm{B} 30$ & Octadecyl-3,5-di-tert-butyl-4-hydroxyhydro cinnamate & $2082-79-3$ & 531 & 530 \\
\hline & & B31 & 1,1,3-Tris(2-methyl-4-hydroxy-5-tert-butylphenyl)butane & $1843-03-4$ & 544 & 339 \\
\hline & & B32 & $\begin{array}{l}\text { 1,3,5-Tris(3,5-di-tert-butyl-4-hydroxybenzyl) } \\
\text {-s-triazine-2,4,6-(1H,3H,5H)-trione }\end{array}$ & $27676-62-6$ & 784 & 219 \\
\hline & & B33 & $\begin{array}{l}\text { 1,3,5-Trimethyl-2,4,6-tris } \\
\text { (3,5-di-tert-butyl-4-hydroxybenzyl)benzene }\end{array}$ & $1709-70-2$ & 775 & 775 \\
\hline & & B34 & $\begin{array}{l}\text { 2,2'-Oxamidobis [ethyl-3- } \\
\text { (3,5-di-tert-butyl-4-hydroxyphenyl)propionate] }\end{array}$ & 70331-94-1 & 697 & 219 \\
\hline & & B35 & $\begin{array}{l}\text { 1,3,5-Tris(4-tert-butyl-3-hydroxy-2,6-dimethylbenzyl) } \\
\text {-1,3,5-triazine-2,4,6-(1H,3H,5H)-trione }\end{array}$ & $40601-76-1$ & 900 & 190 \\
\hline & Sulfurate & B36 & 4,4'-Thiobis(3-methyl-6-tert-butylphenol) & $96-69-5$ & 359 & 358 \\
\hline & & B37 & Didodecyl 3,3'-thiodipropionate & $123-28-4$ & 515 & 55 \\
\hline & & B38 & Di-n-tetradecyl 3,3'-thiobispropionate & $16545-54-3$ & 571 & 57 \\
\hline & & B39 & Dioctadecyl 3,3'-thiodipropionate & 693-36-7 & 683 & 55 \\
\hline & Phosphite & B40 & Tris(2,4-di-tert-butylphenyl)phosphite & $31570-04-4$ & 647 & 441 \\
\hline \multirow{9}{*}{$\begin{array}{l}\text { Ultraviolet } \\
\text { absorber }\end{array}$} & Benzophenone & B41 & 2-Hydroxy-4-methoxybenzophenone & $131-57-7$ & 228 & 227 \\
\hline & & B42 & 2,2'-Dihydroxy-4-methoxybenzophenone & $131-53-3$ & 244 & 244 \\
\hline & & B43 & 2-Hydroxy-4-n-octabenzophenone & $1843-05-6$ & 326 & 213 \\
\hline & & B44 & 2-(2'-Hydroxy-5'-methylphenyl)benzotriazole & $2440-22-4$ & 225 & 225 \\
\hline & Benzotriazole & B45 & $\begin{array}{l}\text { 2-(2'-Hydroxy-3'-tert-butyl-5'-methylphenyl) } \\
\text {-5-chlorobenzotriazole }\end{array}$ & $3896-11-5$ & 316 & 300 \\
\hline & & B46 & 2-(2'-Hydroxy-3',5'-di-tert-amylphenyl) benzotriazole & $25973-55-1$ & 351 & 322 \\
\hline & Other & B47 & 4-tert-Butylphenylsalicylate & $87-18-3$ & 270 & 121 \\
\hline & & B48 & $\begin{array}{l}\text { 2,4-Di-tert-butylphenyl-3,5-di-tert-butyl-4 } \\
\text {-hydroxybenzoate }\end{array}$ & $4221-80-1$ & 439 & 233 \\
\hline & & B49 & 2,5-Di(5-tert-butylbenzoxazol-2-yl)thiophene & $7128-64-5$ & 430 & 415 \\
\hline
\end{tabular}




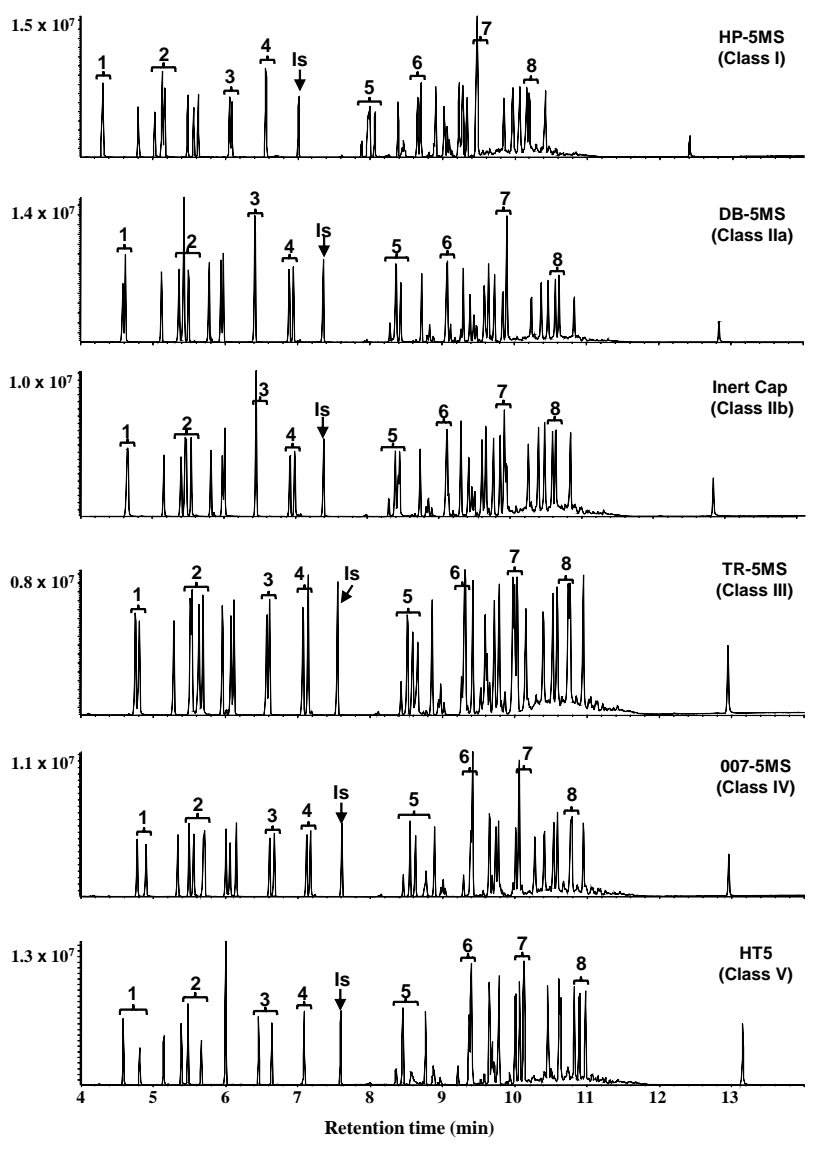

Figure 1. Typical GC/MS total ion chromatograms for test Solution A.

the chromatogram pattern and separation of fifteen peak groups (peak Groups 1-8 in test Solution A, and peak Groups 9-15 in test Solution B) in the analyses of the additives, the fifteen columns were classified into various categories (Classes I-V, Table 4).

Class I contains six columns which are 5\% diphenyl polysiloxane/dimethyl polysiloxane phase type columns. Complete separation of the peaks in Groups 3, 6, 8,10 , and 11 was achieved in the total ion chromatograms of Class I, whereas the peaks in Groups 1, 7, and 15 were not separated. However, there were certain differences in the separation of peak Groups 2, 5, 12, and 14. In particular, among the Class I columns, the chromatogram obtained using the Rxi-5HT column was slightly different from those using other columns in terms of the separation pattern of peak Groups 4 and 9.

The chromatogram patterns obtained using the phenyl-arylene/dimethyl polysiloxane phase type columns were different from those of the other columns, thus, the columns were divided into Classes II to IV. Class II contains five columns. The chromatograms obtained with these columns showed clear separation of the peaks in Groups 4, 8, 9, 10,11,12, and 14, whereas the peaks in Groups 3 and 6 were not separated. The five

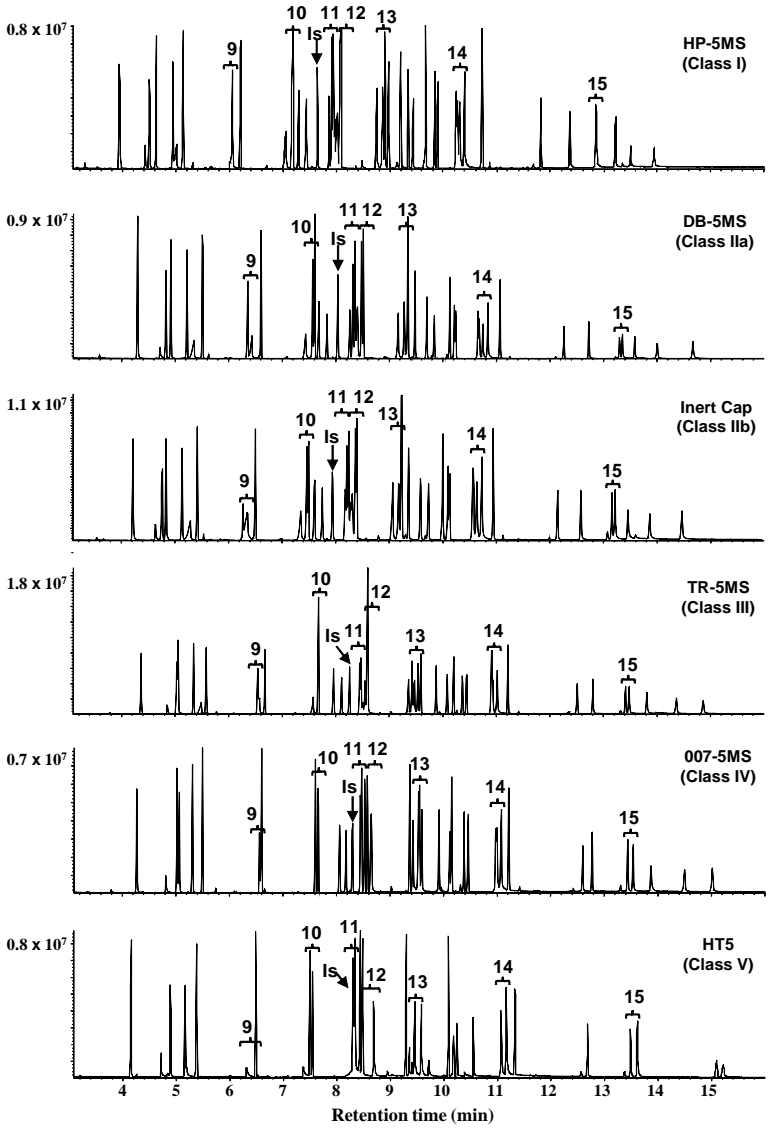

Figure 2. Typical GC/MS total ion chromatograms for test Solution B.

columns in Class II were further classified into Class IIa and Class IIb based on the separation pattern of peak Groups 1, 5, 7, and 14. Class III contained two columns. The TIC showed clear separation of the peaks in Groups 1-5, 7, 8, 11, 13, and 15. However, only the chromatogram pattern obtained using the $007-5 \mathrm{MS}$ column which contains a phenyl-arylene/dimethyl polysiloxane phase, was different from that of Classes II and III. Most of the peaks in the chromatogram of from this column appeared to be sharper than those obtained with the other columns. Furthermore, five additives such as fatty acids (B10-13) and a phenol type antioxidant (B32) could not be detected using the 007-5MS column. Therefore, the 007-5MS column was classified into Class IV.

The HT5 column is a siloxane-carborane phase type column. This column was classified into Class V. The chromatogram pattern obtained with this column was different from those obtained using the other columns. Thirteen kinds of additives, comprising two plasticizers (A11 and A42), six antioxidants (B24, B31, B32, and B34-36), two benzophenone type ultraviolet absorbers (B41 and B43), and three others (A45-A47) could not be detected in the chromatogram. 
Table 4. Column classification and peak separations in respective chromatograms.

\begin{tabular}{|c|c|c|c|c|c|c|c|c|c|c|c|c|c|c|c|c|c|}
\hline \multirow{6}{*}{ Class } & \multirow{6}{*}{ Column } & \multirow{6}{*}{$\begin{array}{l}\text { Phase } \\
\text { type }\end{array}$} & \multicolumn{8}{|c|}{ Peak group in test Solution A } & \multicolumn{7}{|c|}{ Peak group in test Solution B } \\
\hline & & & 1 & 2 & 3 & 4 & 5 & 6 & 7 & 8 & 9 & 10 & 11 & 12 & 13 & 14 & 15 \\
\hline & & & A1 & $\mathrm{A} 2$ & A4 & A16 & A 32 & A19 & A8 & A11 & B11 & B6 & B7 & B1 & B3 & B4 & $\mathrm{B} 30$ \\
\hline & & & A12 & A13 & A 15 & A46 & A33 & A43 & A9 & $\mathrm{A} 20$ & B24 & B17 & B8 & B9 & B19 & B5 & B37 \\
\hline & & & & A36 & & & A34 & & $\mathrm{A} 21$ & A26 & & & B13 & B18 & B25 & B36 & \\
\hline & & & & $\mathrm{A} 42$ & & & A47 & & A22 & A 28 & & & B42 & & & B43 & \\
\hline \multirow[t]{6}{*}{ Class I } & HP-5MS & (a) & $\mathrm{n}$ & $\mathrm{y} / \mathrm{n}$ & $\mathrm{y}$ & $\mathrm{n}$ & $\mathrm{y} / \mathrm{n}$ & $\mathrm{y}$ & $\mathrm{n}$ & $\mathrm{y}$ & $\mathrm{n}$ & y & $\mathrm{y}$ & $\mathrm{y} / \mathrm{n}$ & $y / n$ & y & $\mathrm{n}$ \\
\hline & DB-5 & (a) & $\mathrm{n}$ & $\mathrm{y}$ & y & $\mathrm{n}$ & y & $\mathrm{y}$ & $\mathrm{n}$ & $\mathrm{y}$ & $\mathrm{n}$ & y & $\mathrm{y}$ & $\mathrm{y}$ & $y / n$ & y & $\mathrm{n}$ \\
\hline & ZB-5 & (a) & $\mathrm{n}$ & $y / n$ & $\mathrm{y}$ & $\mathrm{n}$ & $\mathrm{y} / \mathrm{n}$ & y & $\mathrm{n}$ & $\mathrm{y}$ & $\mathrm{n}$ & $\mathrm{y}$ & $\mathrm{y}$ & $\mathrm{y}$ & $\mathrm{y} / \mathrm{n}$ & $\mathrm{y}$ & $\mathrm{n}$ \\
\hline & DB-5HT & (a) & $\mathrm{n}$ & $\mathrm{y}$ & $\mathrm{y}$ & $\mathrm{n}$ & $y / n$ & $\mathrm{y}$ & $\mathrm{n}$ & $\mathrm{y}$ & $\mathrm{n}$ & $\mathrm{y}$ & $\mathrm{y}$ & $\mathrm{y}$ & $\mathrm{y} / \mathrm{n}$ & $\mathrm{y}$ & $\mathrm{n}$ \\
\hline & ZB-5HT & (a) & $\mathrm{n}$ & $y / n$ & $\mathrm{y}$ & $\mathrm{n}$ & $\mathrm{y}$ & $\mathrm{y}$ & $\mathrm{n}$ & $\mathrm{y}$ & $\mathrm{n}$ & y & y & y & $y / n$ & $y / n$ & $\mathrm{n}$ \\
\hline & Rxi-5HT & (a) & $\mathrm{n}$ & $\mathrm{y}$ & $\mathrm{y}$ & $\mathrm{y}$ & y & $\mathrm{y}$ & $\mathrm{n}$ & $\mathrm{y}$ & y & $\mathrm{y}$ & $\mathrm{y}$ & $\mathrm{y}$ & $\mathrm{y} / \mathrm{n}$ & $\mathrm{y} / \mathrm{n}$ & $\mathrm{n}$ \\
\hline \multirow[t]{2}{*}{ Class IIa } & DB-5MS & (b) & $\mathrm{y}$ & $y / n$ & $\mathrm{n}$ & $\mathrm{y}$ & $\mathrm{n}$ & $\mathrm{n}$ & $\mathrm{n}$ & $\mathrm{y}$ & $\mathrm{y}$ & $\mathrm{y}$ & $\mathrm{y}$ & $\mathrm{y}$ & $\mathrm{n}$ & $\mathrm{y}$ & y \\
\hline & Rxi-5 & (b) & $\mathrm{y}$ & $\mathrm{y} / \mathrm{n}$ & $\mathrm{n}$ & $\mathrm{y}$ & $\mathrm{n}$ & $\mathrm{n}$ & $\mathrm{n}$ & $\mathrm{y}$ & $\mathrm{y}$ & $\mathrm{y}$ & y & $\mathrm{y}$ & $\mathrm{n}$ & $\mathrm{y}$ & y \\
\hline \multirow[t]{3}{*}{ Class IIb } & Inert Cap & (b) & $\mathrm{n}$ & $y / n$ & $\mathrm{n}$ & $\mathrm{y}$ & y & $\mathrm{n}$ & $\mathrm{y}$ & $\mathrm{y}$ & $\mathrm{y}$ & y & y & $\mathrm{y}$ & $\mathrm{n}$ & $\mathrm{y} / \mathrm{n}$ & y \\
\hline & CP-Sil & (b) & $\mathrm{n}$ & $\mathrm{y}$ & $\mathrm{n}$ & $\mathrm{y}$ & $\mathrm{y}$ & $\mathrm{n}$ & $\mathrm{y}$ & $\mathrm{y}$ & $\mathrm{y}$ & $\mathrm{y}$ & $\mathrm{y}$ & $\mathrm{y}$ & $\mathrm{y}$ & $\mathrm{y} / \mathrm{n}$ & y \\
\hline & $\mathrm{VF}-5 \mathrm{~ms}$ & (b) & $y / n$ & $\mathrm{y}$ & $\mathrm{n}$ & $\mathrm{y}$ & y & $\mathrm{n}$ & $\mathrm{y}$ & $\mathrm{y}$ & $\mathrm{y}$ & $\mathrm{y}$ & y & $\mathrm{y}$ & $\mathrm{y}$ & $\mathrm{y} / \mathrm{n}$ & $\mathrm{y}$ \\
\hline \multirow[t]{2}{*}{ Class III } & TR-5MS & (b) & $\mathrm{y}$ & $\mathrm{y}$ & $\mathrm{y}$ & $\mathrm{y}$ & $\mathrm{y}$ & $\mathrm{n}$ & $\mathrm{y}$ & $\mathrm{y}$ & $\mathrm{n}$ & $\mathrm{n}$ & $\mathrm{y}$ & $\mathrm{n}$ & $\mathrm{y}$ & $\mathrm{n}$ & y \\
\hline & BPX5 & (b) & $\mathrm{y}$ & $\mathrm{y}$ & $\mathrm{y}$ & $\mathrm{y}$ & $\mathrm{y}$ & $\mathrm{n}$ & $\mathrm{y}$ & $\mathrm{y}$ & $y / n$ & $\mathrm{n}$ & $\mathrm{y}$ & $\mathrm{n}$ & $\mathrm{y}$ & $\mathrm{n}$ & $\mathrm{y}$ \\
\hline Class IV & $007-5 \mathrm{MS}$ & (b) & $\mathrm{y}$ & $\mathrm{y} / \mathrm{n}$ & $\mathrm{y}$ & $\mathrm{y}$ & $\mathrm{y}$ & $y / n$ & $\mathrm{n}$ & $\mathrm{n}$ & * & $\mathrm{y}$ & $\mathrm{y}$ & ${ }^{*}$ & $\mathrm{y}$ & $\mathrm{n}$ & $\mathrm{y}$ \\
\hline Class V & HT5 & (c) & $\mathrm{y}$ & * & $\mathrm{y}$ & * & * & $\mathrm{y}$ & $\mathrm{y} / \mathrm{n}$ & $\mathrm{y}$ & $*$ & $\mathrm{y}$ & $\mathrm{n}$ & $\mathrm{n}$ & $\mathrm{n}$ & * & $\mathrm{y}$ \\
\hline
\end{tabular}

(a): 5\% diphenyl polysiloxane/dimethyl polysiloxane, (b): phenyl-arylene/dimethyl polysiloxane, (c): siloxane-carborane. y: Peaks were separated completely. $\mathrm{y} / \mathrm{n}$ : Peaks were incompletely separated. n: Peaks overlapped completely. ${ }^{*}$ : A portion of peak was not detected.

\subsection{Comparison of Retention Time}

The accurate lengths of all of the columns were determined to be between $15 \mathrm{~m}$ and $16.5 \mathrm{~m}$ (Table 1). The inner diameters and film thickness were not measured; these may vary depending on the column products as well as length. These differences should affect the retention times of the additives. Therefore, the relative retention times (RRTs) of the additives during the analyses using the columns were compared. Dibutylphthalate (A17) and 4-tert-butylphenylsalicylate (B47) are chosen for use as internal standards in test Solutions A and B, respectively, given that these substances were detected in the approximate middle of the additives detected in all of the chromatograms, and did not overlap with the peaks of other additives in the chromatograms. Analysis of the additives using the Inert Cap column were performed using three lots of the column having different serial numbers. The obtained analytical data were used as an index of reproducibility for instrumental conditions, internal standard correction, and lot-to-lot variation.
The RRT of the typical columns and coefficient of variation $(\mathrm{CV})$ using three lots of Inert Cap, Class I, Class II, and all fifteen columns are shown in Tables 5 and 6.

The RRT of the additives were not significantly different. However, in the case of certain additives with similar retention times, the order of detection changed from column to column. The CV of the RRTs of the additives using the three lots of Inert Cap products were $0.1 \%-0.9 \%$, and were mostly below $0.5 \%$. The results suggest that there were no differences in the instrumental conditions of this study. The additives detected in the early stage $(\mathrm{RRT}<0.75)$ and middle stages $(\mathrm{RRT}=0.75$ -2 , approximate retention time of $5.6-15$ minutes) had largely similar RRTs, irrespective of the columns, and their CVs were low $(0.2 \%-1.9 \%)$. However, the RRTs of the additives detected at the later stage (RRT $>2$ ) varied widely. Thus, CVs appear to be high $(3.2 \%-6.3 \%)$. This later stage corresponds largely to the maximum temperature $\left(320^{\circ} \mathrm{C}\right)$ holding period in the column tem- 
Table 5. Relative retention time of additives in test Solution A.

\begin{tabular}{|c|c|c|c|c|c|c|c|c|c|c|}
\hline \multirow{2}{*}{ No. } & \multirow{2}{*}{$\begin{array}{c}\text { Class I } \\
\text { HP-5MS }\end{array}$} & \multirow{2}{*}{$\begin{array}{l}\text { Class IIa } \\
\text { DB-5MS }\end{array}$} & \multirow{2}{*}{$\begin{array}{l}\text { Class IIb } \\
\text { Inert Cap }\end{array}$} & \multirow{2}{*}{$\begin{array}{l}\text { Class III } \\
\text { TR-5MS }\end{array}$} & \multirow{2}{*}{$\begin{array}{l}\text { Class IV } \\
007-5 \mathrm{MS}\end{array}$} & \multirow{2}{*}{$\begin{array}{c}\text { Class V } \\
\text { HT5 }\end{array}$} & \multicolumn{4}{|c|}{ CV (\%) } \\
\hline & & & & & & & Inert Cap 3 lots & Class I & Class II & All column \\
\hline A17 & 7.01 & 7.37 & 7.22 & 7.56 & 7.61 & 7.60 & - & - & - & - \\
\hline A12 & 0.61 & 0.62 & 0.62 & $0.64^{*}$ & $0.64^{*}$ & $0.63^{*}$ & 0.2 & 0.9 & 0.6 & 1.5 \\
\hline A1 & 0.61 & 0.63 & 0.62 & $0.63^{*}$ & $0.63^{*}$ & $0.60^{*}$ & 0.2 & 0.9 & 0.7 & 1.2 \\
\hline A40 & 0.68 & 0.69 & 0.69 & 0.70 & 0.70 & 0.68 & 0.2 & 0.6 & 0.5 & 0.9 \\
\hline $\mathrm{A} 2$ & 0.72 & 0.73 & 0.72 & 0.73 & $0.73^{*}$ & 0.71 & 0.2 & 0.6 & 0.4 & 0.8 \\
\hline A36 & 0.73 & 0.74 & 0.73 & 0.73 & $0.72^{*}$ & 0.72 & 0.2 & 0.6 & 0.6 & 1.0 \\
\hline A13 & 0.73 & 0.74 & 0.73 & 0.75 & $0.75^{*}$ & 0.75 & 0.2 & 0.6 & 0.4 & 0.8 \\
\hline A42 & 0.74 & 0.74 & 0.74 & 0.75 & $0.75^{*}$ & ND & 0.2 & 0.7 & 0.4 & 0.7 \\
\hline A14 & 0.78 & 0.78 & 0.78 & 0.79 & 0.79 & 0.79 & 0.2 & 0.4 & 0.3 & 0.5 \\
\hline A45 & 0.79 & 0.81 & 0.80 & 0.80 & 0.80 & ND & 0.2 & 0.5 & 0.3 & 0.4 \\
\hline A3 & 0.80 & 0.81 & 0.81 & 0.81 & 0.81 & 0.79 & 0.2 & 0.3 & 0.2 & 0.6 \\
\hline A15 & 0.87 & 0.87 & 0.87 & 0.87 & $0.88^{*}$ & $0.87^{*}$ & 0.2 & 0.2 & 0.1 & 0.3 \\
\hline A4 & 0.86 & 0.87 & 0.87 & 0.87 & $0.87^{*}$ & $0.85^{*}$ & 0.2 & 0.2 & 0.2 & 0.6 \\
\hline A16 & 0.93 & 0.93 & 0.93 & 0.94 & 0.93 & 0.93 & 0.2 & 0.1 & $<0.1$ & 0.2 \\
\hline A46 & 0.94 & 0.94 & 0.94 & 0.95 & 0.94 & ND & 0.2 & 0.3 & $<0.1$ & 0.3 \\
\hline A5 (m) & 1.13 & 1.13 & 1.13 & 1.12 & 1.11 & 1.10 & 0.2 & 0.3 & 0.2 & 0.7 \\
\hline A34 & 1.14 & 1.14 & 1.14 & 1.13 & 1.12 & 1.11 & 0.2 & 0.3 & $<0.1$ & 0.7 \\
\hline A47 & 1.14 & 1.14 & 1.14 & $1.15^{*}$ & $1.15^{*}$ & ND & 0.2 & 0.3 & 0.2 & 0.5 \\
\hline A32 & 1.15 & 1.15 & 1.15 & $1.14^{*}$ & $1.13^{*}$ & 1.13 & 0.2 & 0.3 & 0.1 & 0.6 \\
\hline A33 & 1.20 & 1.19 & 1.19 & 1.17 & 1.17 & 1.15 & 0.2 & 0.4 & 0.2 & 1.4 \\
\hline A37 & 1.21 & 1.21 & 1.20 & 1.19 & 1.18 & 1.17 & 0.2 & 0.4 & 0.2 & 0.9 \\
\hline A38 & 1.24 & 1.24 & 1.24 & 1.23 & 1.23 & 1.23 & 0.2 & 0.3 & 0.1 & 0.4 \\
\hline A18 & 1.24 & 1.24 & 1.24 & 1.23 & 1.24 & 1.24 & 0.3 & 0.3 & 0.2 & 0.3 \\
\hline A6 & 1.27 & 1.27 & 1.27 & 1.25 & 1.24 & 1.24 & 0.2 & 0.5 & 0.2 & 0.9 \\
\hline A43 & 1.29 & 1.28 & 1.28 & 1.27 & 1.27 & 1.27 & 0.2 & 0.4 & 0.2 & 0.5 \\
\hline A19 (m) & 1.29 & 1.29 & 1.29 & 1.27 & 1.27 & $1.28^{*}$ & 0.2 & 0.4 & 0.2 & 0.6 \\
\hline A39 & 1.32 & 1.31 & 1.30 & 1.29 & 1.28 & $1.27^{*}$ & 0.3 & 0.4 & 0.3 & 1.0 \\
\hline A44 (m) & 1.32 & 1.31 & 1.31 & $1.31^{*}$ & $1.31^{*}$ & $1.31^{*}$ & 0.3 & 0.4 & 0.2 & 0.4 \\
\hline A7 (m) & 1.32 & 1.32 & $1.32^{*}$ & $1.30^{*}$ & $1.29^{*}$ & $1.28^{*}$ & 0.2 & 0.8 & 0.2 & 1.1 \\
\hline A 20 & 1.32 & 1.32 & $1.31^{*}$ & $1.29^{*}$ & $1.28^{*}$ & $1.29^{*}$ & 0.2 & 0.5 & 0.3 & 1.0 \\
\hline A21 & 1.33 & 1.33 & 1.33 & 1.32 & 1.32 & $1.33^{*}$ & 0.2 & 0.4 & 0.2 & 0.4 \\
\hline A9 & 1.35 & 1.35 & $1.35^{*}$ & $1.34^{*}$ & $1.35^{*}$ & $1.33^{*}$ & 0.2 & 0.5 & 0.2 & 0.6 \\
\hline A8 & 1.35 & 1.35 & $1.35^{*}$ & $1.33^{*}$ & $1.32^{*}$ & $1.32^{*}$ & 0.2 & 0.5 & 0.3 & 0.9 \\
\hline A22 & 1.35 & 1.35 & $1.34^{*}$ & $1.32^{*}$ & $1.32^{*}$ & 1.33 & 0.3 & 0.7 & 0.2 & 0.9 \\
\hline A23 (m) & 1.35 & 1.40 & 1.36 & 1.36 & $1.34^{*}$ & 1.33 & 0.2 & 1.0 & 1.1 & 1.6 \\
\hline A24 (m) & 1.41 & 1.40 & 1.39 & 1.37 & 1.37 & 1.38 & 0.2 & 0.5 & 0.3 & 0.8 \\
\hline $\mathrm{A} 10(\mathrm{~m})$ & 1.42 & 1.40 & 1.40 & 1.39 & 1.38 & 1.38 & 0.2 & 0.8 & 0.2 & 0.9 \\
\hline A25 & 1.42 & 1.42 & 1.42 & 1.39 & 1.39 & 1.40 & 0.2 & 0.6 & 0.3 & 0.9 \\
\hline A41 & 1.44 & 1.44 & 1.43 & 1.40 & 1.39 & 1.40 & 0.2 & 0.6 & 0.3 & 1.1 \\
\hline A26 & 1.45 & 1.45 & 1.44 & 1.42 & 1.42 & 1.42 & 0.2 & 0.6 & 0.4 & 0.8 \\
\hline A27 & 1.46 & 1.46 & 1.45 & 1.42 & 1.42 & 1.43 & 0.2 & 0.6 & 0.4 & 0.9 \\
\hline A28 & 1.47 & 1.47 & 1.45 & 1.44 & 1.43 & 1.44 & 0.4 & 0.8 & 0.6 & 1.0 \\
\hline A11 (m) & 1.48 & 1.47 & 1.46 & 1.45 & 1.43 & ND & 0.5 & 1.0 & 0.5 & 1.1 \\
\hline A35 & 1.49 & 1.49 & 1.48 & 1.45 & 1.44 & 1.44 & 0.2 & 0.6 & 0.4 & 1.1 \\
\hline A29 (m) & 1.48 & 1.51 & 1.49 & 1.47 & 1.45 & 1.48 & 0.3 & 0.5 & 0.6 & 1.0 \\
\hline A30 (m) & 1.54 & 1.55 & 1.54 & 1.51 & 1.50 & 1.50 & 0.7 & 1.0 & 0.8 & 1.3 \\
\hline A31 & 1.77 & 1.79 & 1.75 & 1.71 & 1.70 & 1.73 & 0.2 & 0.8 & 0.7 & 1.3 \\
\hline
\end{tabular}

A17 was used as an internal standard, the values show retention times (min). Each value is the average of three analytical runs. ND: not detected. (m): Additive was detected as multi-peaks on chromatogram; the values were calculated from the maximum peak. *: The order of detection differs from the Class I columns. 
Table 6. Relative retention time of additives in test Solution B.

\begin{tabular}{|c|c|c|c|c|c|c|c|c|c|c|}
\hline \multirow[t]{2}{*}{ No. } & \multirow{2}{*}{$\begin{array}{l}\text { Class I } \\
\text { HP-5MS }\end{array}$} & \multirow{2}{*}{$\begin{array}{l}\text { Class IIa } \\
\text { DB-5MS }\end{array}$} & \multirow{2}{*}{$\begin{array}{l}\text { Class IIb } \\
\text { Inert Cap }\end{array}$} & \multirow{2}{*}{$\begin{array}{l}\text { Class III } \\
\text { TR-5MS }\end{array}$} & \multirow{2}{*}{$\begin{array}{l}\text { Class IV } \\
007-5 \mathrm{MS}\end{array}$} & \multirow{2}{*}{$\begin{array}{c}\text { Class V } \\
\text { HT5 }\end{array}$} & \multicolumn{4}{|c|}{ CV (\%) } \\
\hline & & & & & & & Inert Cap 3 lots & Class I & Class II & All column \\
\hline B47 & 7.38 & 7.68 & 7.62 & 7.96 & 8.06 & 8.34 & - & - & - & - \\
\hline B14 & 0.52 & 0.53 & 0.53 & 0.53 & 0.52 & 0.50 & 0.3 & 1.1 & 1.4 & 1.9 \\
\hline B21 & 0.59 & 0.60 & 0.60 & 0.61 & 0.61 & 0.57 & 0.2 & 0.7 & 0.8 & 1.8 \\
\hline B22 & 0.61 & 0.61 & 0.61 & 0.61 & 0.61 & 0.59 & 0.2 & 0.8 & 0.9 & 1.5 \\
\hline B23 & 0.65 & 0.65 & 0.65 & 0.65 & 0.64 & 0.62 & $<0.1$ & 0.6 & 0.7 & 1.6 \\
\hline B10 & 0.66 & 0.67 & 0.66 & 0.66 & ND & 0.62 & 0.1 & 0.5 & 0.5 & 1.7 \\
\hline B15 & 0.67 & 0.69 & 0.68 & 0.68 & 0.66 & 0.65 & 0.1 & 0.8 & 0.7 & 1.6 \\
\hline B11 & 0.79 & $0.80^{*}$ & $0.80^{*}$ & $0.80^{*}$ & ND & 0.76 & $<0.1$ & 0.4 & 0.3 & 1.4 \\
\hline B24 & 0.79 & $0.79^{*}$ & $0.79^{*}$ & $0.79^{*}$ & 0.79 & ND & $<0.1$ & 0.2 & 0.3 & 0.5 \\
\hline B16 & 0.81 & 0.82 & 0.82 & 0.81 & 0.80 & 0.78 & $<0.1$ & 0.2 & 0.4 & 1.4 \\
\hline B12 & 0.92 & 0.93 & 0.92 & 0.92 & ND & 0.88 & 0.2 & 0.2 & 0.1 & 1.1 \\
\hline B6 & 0.94 & 0.94 & 0.94 & 0.93 & 0.92 & $0.91^{*}$ & $<0.1$ & $<0.1$ & 0.2 & 1.0 \\
\hline B17 & 0.94 & 0.95 & 0.94 & 0.93 & 0.92 & $0.90^{*}$ & $<0.1$ & $<0.1$ & 0.3 & 1.3 \\
\hline B41 & 0.95 & 0.96 & 0.96 & 0.96 & 0.97 & ND & $<0.1$ & 0.1 & 0.2 & 0.6 \\
\hline B44 & 0.97 & 0.98 & 0.98 & 0.98 & 0.99 & 1.00 & 0.1 & $<0.1$ & 0.1 & 0.7 \\
\hline B42 & 1.03 & 1.03 & 1.03 & $1.04^{*}$ & $1.04^{*}$ & $1.08^{*}$ & 0.1 & $<0.1$ & 0.2 & 1.4 \\
\hline B7 & 1.03 & 1.03 & 1.03 & $1.02^{*}$ & $1.02^{*}$ & $1.00^{*}$ & 0.1 & 0.2 & 0.1 & 1.0 \\
\hline B8 & 1.04 & 1.04 & 1.04 & $1.03^{*}$ & $1.02^{*}$ & $1.00^{*}$ & $<0.1$ & 0.1 & 0.2 & 1.0 \\
\hline B13 & 1.04 & 1.04 & 1.04 & $1.03^{*}$ & $\mathrm{ND}$ & $1.00^{*}$ & 0.1 & 0.1 & 0.2 & 1.0 \\
\hline B1 & 1.05 & 1.05 & 1.05 & 1.04 & $1.04^{*}$ & $1.04^{*}$ & 0.2 & 0.2 & 0.1 & 0.3 \\
\hline B9 & 1.05 & 1.05 & 1.05 & 1.04 & $1.03^{*}$ & $1.02^{*}$ & 0.1 & 0.2 & 0.2 & 1.0 \\
\hline B18 & 1.06 & 1.06 & 1.06 & 1.04 & $1.03^{*}$ & $1.01^{*}$ & 0.1 & 0.2 & 0.3 & 1.3 \\
\hline B2 & 1.14 & 1.14 & 1.14 & 1.13 & $1.14^{*}$ & 1.13 & 0.2 & 0.3 & 0.2 & 0.3 \\
\hline B3 & 1.16 & 1.15 & 1.16 & $1.15^{*}$ & $1.15^{*}$ & $1.15^{*}$ & 0.1 & 0.4 & 0.3 & 0.4 \\
\hline B19 & 1.16 & 1.16 & 1.16 & $1.14^{*}$ & $1.13^{*}$ & $1.13^{*}$ & $<0.1$ & 0.4 & 0.3 & 1.1 \\
\hline B25 & 1.17 & 1.16 & 1.16 & 1.15 & $1.16^{*}$ & $1.12^{*}$ & $<0.1$ & 0.5 & 0.2 & 1.1 \\
\hline B26 & 1.20 & 1.18 & 1.18 & 1.16 & $1.15^{*}$ & $1.12^{*}$ & 0.2 & 0.3 & 0.2 & 1.8 \\
\hline B27 & 1.22 & 1.21 & 1.21 & 1.20 & 1.20 & 1.17 & 0.2 & 0.4 & 0.2 & 1.1 \\
\hline B45 & 1.23 & 1.22 & 1.23 & 1.22 & 1.22 & $1.23^{*}$ & 0.2 & 0.3 & 0.3 & 0.4 \\
\hline B20 & 1.26 & 1.26 & 1.26 & 1.24 & 1.22 & $1.21^{*}$ & 0.2 & 0.4 & 0.3 & 1.3 \\
\hline B46 & 1.28 & 1.27 & $1.28^{*}$ & $1.27^{*}$ & $1.26^{*}$ & $1.27^{*}$ & 0.2 & 0.4 & 0.3 & 0.5 \\
\hline B28 & 1.29 & 1.27 & $1.27^{*}$ & $1.26^{*}$ & $1.25^{*}$ & $1.22^{*}$ & 0.2 & 0.4 & 0.3 & 1.3 \\
\hline B36 & 1.34 & 1.33 & 1.33 & 1.32 & 1.32 & $\mathrm{ND}$ & 0.2 & 0.4 & 0.4 & 0.5 \\
\hline B43 & 1.34 & 1.33 & 1.33 & 1.32 & 1.32 & ND & 0.2 & 0.4 & 0.3 & 0.5 \\
\hline B4 & 1.34 & 1.34 & 1.34 & 1.32 & 1.33 & 1.33 & 0.2 & 0.4 & 0.3 & 0.6 \\
\hline B5 & 1.36 & 1.35 & 1.35 & 1.33 & 1.34 & 1.34 & 0.2 & 0.5 & 0.4 & 0.6 \\
\hline B48 & 1.40 & 1.38 & 1.38 & 1.36 & 1.35 & 1.36 & 0.2 & 0.5 & 0.4 & 1.0 \\
\hline B29 & 1.54 & 1.52 & 1.53 & 1.52 & 1.52 & 1.51 & 0.6 & 0.5 & 0.5 & 0.7 \\
\hline B40 & 1.61 & 1.58 & 1.58 & 1.55 & 1.54 & 1.52 & 0.2 & 0.6 & 0.5 & 1.6 \\
\hline B30 & 1.68 & 1.65 & 1.66 & 1.62 & 1.62 & 1.62 & 0.3 & 0.7 & 0.5 & 1.1 \\
\hline B37 & 1.68 & 1.66 & 1.66 & 1.63 & 1.63 & 1.63 & 0.3 & 0.7 & 0.5 & 1.0 \\
\hline B31 & 1.72 & 1.69 & 1.69 & 1.67 & 1.67 & ND & 0.3 & 0.7 & 0.5 & 0.9 \\
\hline B49 & 1.76 & 1.74 & 1.75 & 1.74 & 1.75 & 1.81 & 0.3 & 0.5 & 0.4 & 1.0 \\
\hline B38 & 1.82 & 1.82 & 1.82 & 1.80 & 1.81 & 1.83 & 0.3 & 0.6 & 0.4 & 0.6 \\
\hline B32 & 2.41 & 2.45 & 2.41 & 2.50 & ND & ND & 0.5 & 3.0 & 2.5 & 3.2 \\
\hline B34 & 2.43 & 2.55 & 2.52 & $2.64^{*}$ & $2.81^{*}$ & ND & 0.5 & 3.2 & 2.9 & 5.0 \\
\hline B39 & 2.47 & $2.69^{*}$ & $2.65^{*}$ & $2.72^{*}$ & $2.85^{*}$ & $3.00^{*}$ & 0.3 & 3.4 & 3.9 & 6.3 \\
\hline B33 & 2.50 & $2.55^{*}$ & $2.52^{*}$ & $2.60^{*}$ & $2.66^{*}$ & $2.75^{*}$ & 0.9 & 3.3 & 2.8 & 4.1 \\
\hline B35 & 2.73 & 2.86 & 2.83 & 3.06 & 3.25 & ND & 0.6 & 3.8 & 3.4 & 6.3 \\
\hline
\end{tabular}

B47 was used as an internal standard, the values show retention times (min). Each value is the average of three analytical runs. ND: not detected. ${ }^{*}$ The order of detection differs from the Class I columns. 
perature control program. These results suggest that the RRTs of the additives detected during the heat-up period in the column temperature control program were similar for all columns, however, the RRTs of the additives detected during the holding period varied depending on the column.

The CVs of the RRTs of the additives assayed using the Classes I and II columns were significantly lower than those of all fifteen columns. In particular, the CVs of the additives detected in the middle stage ( $R R T=0.75$ -2) were similar to those obtained using the three lots of Inert Cap. Slight variation of the RTTs and the order of detection of certain additives were observed using the Classes III-V columns. Specifically, some additives were not detected on the 007-5MS and HT5 columns.

Given that the RRT of most of the additives was similar in the analyses using the fifteen columns, the properties of these columns are deemed equivalent in terms of the retention time of the additives. Therefore, for identification assay via GC analysis, these columns are deemed to be compatible with each other. Specifically, the eleven kinds of columns, classified into Classes I and II, exhibit largely equivalent performance. On the other hand, for analysis of the additives using the four kinds of columns classified in Classes III-V, the column identity might be important in terms of the detection order, and the physiochemical properties or structure of the analytes.

\subsection{Comparison of Sensitivity}

The detection sensitivity of the columns for the additives was analyzed based on the relative peak areas (RPAs) of the additives to the peak area of the internal standard. However, the data for twenty-two additives could not be compared because their RPA was small (less than 0.1) and because some additives were detected as multi-peaks. The RPAs and corresponding CVs for each additive are shown in Tables $\mathbf{7}$ and $\mathbf{8}$. To determine the effects of the inevitable basic conditions of the instrument, such as contamination in the injection port or ion source, column fixing or MSD tuning, etc., the analyses were performed using three lots of Inert Cap columns as well as performing the retention time comparisons.

In the case of the three lots of Inert Cap columns, the CVs of the RPA were less than $10 \%$. However, for the analyses of the thirteen kinds of additives, such as A25, A27, A31, B4, B10, B11, B17, B19, B22, B24, B25, B28, and $\mathrm{B} 40$, the CVs were larger than $10 \%$. The lubricants and antioxidants were strongly influenced by the inevitable basic conditions of the instrument.

The CVs for the analyses of the additives using all columns were mostly below $20 \%$. However, the CVs for fourteen additives, such as A25, A31, A45, B4, B22, B23, B27, B28, B36, B37, B40, B42, B43, and B46, were larger than $20 \%$, and these additives were mainly antioxi- dants or ultraviolet absorbers. The sensitivities towards these additives varied based on the type of column. The RPAs of a number of additives obtained using the 007-5MS and HT5 columns were significantly different from those obtained with other columns.

For the analysis using the HT5 column, the RPAs of nine plasticizers (A2, A4, A8, A12, A13, A32, A33, A34, and A43) were significantly lower than that achieved with the other columns, although the RPA of A25 was relatively high. The RPA of the amide type lubricants (B1-B5) were relatively low when Class IIb columns were used, but were notably high for the analyses using the 007-5MS and HT5 columns. Furthermore, the RPAs of the hydrocarbons (B14-20) were slightly high when the 007-5MS column was used for analysis. Most of the antioxidants and ultraviolet absorbers were detected at low levels using HT5. Although the RPAs of the antioxidant B37 were high when the DB-5HT and ZB-5HT columns were used, these values were low when the Rxi-5, Inert Cap, CP-Sil, VF-5ms, and TR-5MS columns were used. The RPAs of six antioxidants (B25-28, B31, and B40) and an ultraviolet absorber B41 were high using the DB-5HT and ZB-5HT columns.

The maximum/minimum ratio (Max/Min) of the RPAs was less than 2 for the plasticizers and lubricants, except for the fatty acids (B10-12). Therefore, the limit of determination or detection for these additives using an internal standard method would be similar, even if the analyses were performed using columns other than those specified in the published analytical methods. However, the Max/Min of the antioxidants and ultraviolet absorbers were 1.8-4.7, thus, the sensitivity may vary depending on the columns.

\section{Conclusions}

In this study, ninety-six kinds of additives used for food contact plastics, which have various chemical properties, were analyzed by GC/MS using fifteen kinds of nonpolar capillary columns. These columns were categorized into five classes based on their chromatogram patterns and the degree of peak separation. This study suggests that the performance of most non-polar capillary columns is equivalent. Therefore, it is implied that all of the columns used in this study may be applicable in the identification of the additives used in food contact plastics. However, the RRTs of additives detected during the holding period of the column temperature control program varied depending on the type of column.

The detection sensitivity for certain antioxidants or ultraviolet absorbers may vary depending on the type of column used in the analysis. Thus, it is suggested that the sensitivities or limits of detection should be confirmed in the analysis of these additives. Moreover, certain additives such as fatty acids and some antioxidants, two plas- 
Table 7. Relative peak areas of additives in test Solution A.

\begin{tabular}{|c|c|c|c|c|c|c|c|c|c|c|c|c|c|c|c|c|c|c|c|}
\hline \multirow{2}{*}{ Application } & \multirow{2}{*}{ No. - } & \multicolumn{6}{|c|}{ Class I } & \multicolumn{5}{|c|}{ Class II } & \multicolumn{2}{|c|}{ Class III } & \multirow{2}{*}{$\begin{array}{c}\text { Class IV } \\
007-5 \\
\text { MS }\end{array}$} & \multirow{2}{*}{$\begin{array}{c}\text { Class V } \\
\text { HT5 }\end{array}$} & \multicolumn{2}{|c|}{ CV (\%) } & \multirow{2}{*}{ Max/Min } \\
\hline & & $\begin{array}{l}\text { HP-5 } \\
\text { MS }\end{array}$ & DB-5 & ZB-5 & $\begin{array}{c}\text { DB-5 } \\
\text { HT }\end{array}$ & $\begin{array}{c}\text { ZB-5 } \\
\text { HT }\end{array}$ & $\begin{array}{c}\text { Rxi-5 } \\
\text { HT }\end{array}$ & $\begin{array}{l}\text { DB-5 } \\
\text { MS }\end{array}$ & Rxi-5 & $\begin{array}{l}\text { Inert } \\
\text { Cap }\end{array}$ & CP-Sil & $\begin{array}{c}\mathrm{VF}-5 \\
\mathrm{~ms}\end{array}$ & $\begin{array}{l}\text { TR-5 } \\
\text { MS }\end{array}$ & BPX-5 & & & $\begin{array}{l}\text { Inert Cap } \\
3 \text { lots }\end{array}$ & $\begin{array}{c}\text { All } \\
\text { column }\end{array}$ & \\
\hline Is & & & $5.2 \times 10^{6}$ & & $5.5 \times 10^{6}$ & & $4.6 \times 10^{6}$ & & $3.4 \times 10^{6}$ & & $4.7 \times 10^{6}$ & & $5.4 \times 10^{6}$ & & $3.6 \times 10^{6}$ & & - & - & - \\
\hline \multirow[t]{30}{*}{ Plasticizer } & $\mathrm{A} 1$ & 0.25 & 0.35 & 0.31 & 0.25 & 0.25 & 0.27 & 0.29 & 0.34 & 0.31 & 0.31 & 0.30 & 0.29 & 0.31 & 0.29 & 0.24 & 4 & 12 & 1.5 \\
\hline & $\mathrm{A} 2$ & 0.17 & 0.22 & 0.22 & 0.19 & 0.18 & 0.19 & 0.20 & 0.22 & 0.21 & 0.20 & 0.20 & 0.21 & 0.20 & 0.21 & 0.13 & 4 & 11 & 1.7 \\
\hline & $\mathrm{A} 3$ & 0.27 & 0.36 & 0.30 & 0.28 & 0.24 & 0.28 & 0.33 & 0.38 & 0.35 & 0.35 & 0.28 & 0.28 & 0.34 & 0.30 & 0.27 & 2 & 13 & 1.6 \\
\hline & A4 & 0.16 & 0.19 & 0.20 & 0.18 & 0.17 & 0.17 & 0.18 & 0.19 & 0.18 & 0.18 & 0.18 & 0.18 & 0.18 & 0.18 & 0.12 & 2 & 9 & 1.7 \\
\hline & A6 & 0.32 & 0.37 & 0.31 & 0.34 & 0.35 & 0.33 & 0.40 & 0.36 & 0.39 & 0.37 & 0.31 & 0.30 & 0.36 & 0.34 & 0.39 & 3 & 9 & 1.3 \\
\hline & A8 & 0.19 & 0.15 & 0.13 & 0.18 & 0.19 & 0.15 & 0.18 & 0.15 & 0.15 & 0.15 & 0.13 & 0.15 & 0.16 & 0.15 & 0.11 & 4 & 15 & 1.6 \\
\hline & A9 & 0.61 & 0.44 & 0.45 & 0.60 & 0.63 & 0.51 & 0.47 & 0.45 & 0.47 & 0.47 & 0.48 & 0.49 & 0.47 & 0.51 & 0.42 & 3 & 13 & 1.5 \\
\hline & $\mathrm{A} 12$ & 0.53 & 0.62 & 0.64 & 0.56 & 0.53 & 0.55 & 0.58 & 0.63 & 0.58 & 0.54 & 0.61 & 0.66 & 0.58 & 0.60 & 0.41 & 6 & 10 & 1.5 \\
\hline & A13 & 0.57 & 0.62 & 0.68 & 0.57 & 0.54 & 0.57 & 0.57 & 0.63 & 0.59 & 0.58 & 0.59 & 0.65 & 0.57 & 0.62 & 0.47 & 5 & 8 & 1.4 \\
\hline & A14 & 0.75 & 0.84 & 0.85 & 0.75 & 0.71 & 0.77 & 0.79 & 0.84 & 0.83 & 0.80 & 0.76 & 0.80 & 0.76 & 0.79 & 0.70 & 4 & 6 & 1.2 \\
\hline & A15 & 0.79 & 0.85 & 0.88 & 0.80 & 0.76 & 0.80 & 0.81 & 0.87 & 0.85 & 0.83 & 0.83 & 0.83 & 0.84 & 0.83 & 0.75 & 3 & 4 & 1.2 \\
\hline & A16 & 0.81 & 0.85 & 0.80 & 0.79 & 0.78 & 0.79 & 0.79 & 0.80 & 0.84 & 0.81 & 0.78 & 0.80 & 0.81 & 0.82 & 0.74 & 1 & 3 & 1.1 \\
\hline & $\mathrm{A} 18$ & 0.40 & 0.40 & 0.38 & 0.43 & 0.45 & 0.43 & 0.41 & 0.39 & 0.43 & 0.41 & 0.39 & 0.43 & 0.40 & 0.45 & 0.48 & 2 & 6 & 1.3 \\
\hline & $\mathrm{A} 20$ & 0.28 & 0.29 & 0.25 & 0.30 & 0.29 & 0.28 & 0.29 & 0.28 & 0.30 & 0.29 & 0.26 & 0.25 & 0.28 & 0.27 & 0.28 & 3 & 5 & 1.2 \\
\hline & $\mathrm{A} 21$ & 0.68 & 0.67 & 0.65 & 0.72 & 0.73 & 0.69 & 0.73 & 0.64 & 0.76 & 0.72 & 0.64 & 0.74 & 0.74 & 0.67 & 0.68 & 5 & 6 & 1.2 \\
\hline & $\mathrm{A} 25$ & 0.16 & 0.15 & 0.13 & 0.17 & 0.17 & 0.16 & 0.20 & 0.16 & 0.20 & 0.19 & 0.13 & 0.13 & 0.17 & 0.14 & 0.27 & 11 & 22 & 2.0 \\
\hline & A26 & 1.09 & 0.90 & 0.86 & 1.11 & 1.12 & 1.04 & 1.10 & 0.94 & 1.09 & 1.07 & 0.89 & 0.91 & 1.02 & 1.14 & 1.05 & 7 & 9 & 1.3 \\
\hline & $\mathrm{A} 27$ & 0.18 & 0.17 & 0.15 & 0.19 & 0.19 & 0.18 & 0.23 & 0.18 & 0.24 & 0.22 & 0.15 & 0.15 & 0.21 & 0.17 & 0.20 & 12 & 15 & 1.6 \\
\hline & A31 & 0.24 & 0.13 & 0.17 & 0.28 & 0.30 & 0.22 & 0.28 & 0.18 & 0.26 & 0.25 & 0.16 & 0.20 & 0.20 & 0.20 & 0.20 & 20 & 22 & 2.2 \\
\hline & $\mathrm{A} 32$ & 0.34 & 0.38 & 0.38 & 0.42 & 0.41 & 0.32 & 0.36 & 0.37 & 0.38 & 0.36 & 0.38 & 0.36 & 0.38 & 0.40 & 0.17 & 3 & 16 & 2.5 \\
\hline & $\mathrm{A} 33$ & 0.34 & 0.33 & 0.33 & 0.37 & 0.37 & 0.33 & 0.33 & 0.33 & 0.35 & 0.33 & 0.31 & 0.34 & 0.34 & 0.36 & 0.26 & 2 & 8 & 1.4 \\
\hline & A34 & 0.15 & 0.15 & 0.13 & 0.15 & 0.15 & 0.13 & 0.15 & 0.15 & 0.14 & 0.13 & 0.12 & 0.14 & 0.14 & 0.14 & 0.09 & 1 & 12 & 1.7 \\
\hline & A 35 & 0.41 & 0.32 & 0.32 & 0.42 & 0.43 & 0.35 & 0.39 & 0.33 & 0.41 & 0.38 & 0.30 & 0.32 & 0.35 & 0.34 & 0.31 & 10 & 12 & 1.4 \\
\hline & A36 & 0.65 & 0.77 & 0.76 & 0.64 & 0.58 & 0.68 & 0.71 & 0.74 & 0.72 & 0.71 & 0.69 & 0.69 & 0.70 & 0.71 & 0.67 & 1 & 7 & 1.3 \\
\hline & A 38 & 1.01 & 1.03 & 0.98 & 1.07 & 1.08 & 1.04 & 1.04 & 1.03 & 1.07 & 1.04 & 1.03 & 0.96 & 1.03 & 1.01 & 1.05 & 2 & 3 & 1.1 \\
\hline & A39 & 0.32 & 0.27 & 0.27 & 0.33 & 0.33 & 0.28 & 0.30 & 0.27 & 0.29 & 0.27 & 0.27 & 0.27 & 0.27 & 0.30 & 0.23 & 3 & 9 & 1.2 \\
\hline & $\mathrm{A} 40$ & 0.37 & 0.46 & 0.49 & 0.39 & 0.37 & 0.42 & 0.40 & 0.48 & 0.43 & 0.44 & 0.46 & 0.45 & 0.44 & 0.46 & 0.36 & 3 & 9 & 1.4 \\
\hline & A41 & 0.39 & 0.33 & 0.32 & 0.41 & 0.41 & 0.35 & 0.41 & 0.33 & 0.39 & 0.37 & 0.32 & 0.32 & 0.35 & 0.35 & 0.31 & 8 & 11 & 1.3 \\
\hline & $\mathrm{A} 42$ & 0.63 & 0.90 & 0.84 & 0.78 & 0.71 & 0.75 & 0.83 & 0.89 & 0.87 & 0.86 & 0.82 & 0.81 & 0.82 & 0.76 & ND & 6 & 9 & - \\
\hline & $\mathrm{A} 43$ & 0.39 & 0.39 & 0.35 & 0.43 & 0.44 & 0.36 & 0.39 & 0.38 & 0.39 & 0.36 & 0.35 & 0.39 & 0.38 & 0.39 & 0.28 & 3 & 10 & 1.6 \\
\hline \multirow[t]{3}{*}{ Other } & A45 & 0.12 & 0.15 & 0.16 & 0.15 & 0.14 & 0.29 & 0.17 & 0.17 & 0.16 & 0.15 & 0.14 & 0.13 & 0.15 & 0.14 & ND & 7 & 25 & - \\
\hline & A46 & 1.01 & 1.11 & 1.09 & 1.22 & 1.20 & 1.10 & 1.06 & 1.11 & 1.10 & 1.11 & 1.09 & 1.09 & 1.05 & 1.02 & ND & 4 & 5 & - \\
\hline & A47 & 0.42 & 0.32 & 0.42 & 0.47 & 0.41 & 0.42 & 0.37 & 0.35 & 0.39 & 0.38 & 0.40 & 0.41 & 0.33 & 0.27 & ND & 10 & 13 & - \\
\hline
\end{tabular}

A17 was used as an internal standard (Is), the values show peak areas. Each value is the average of three analytical runs. ND: not detected. 
Table 8. Relative peak areas of additives in test Solution B.

\begin{tabular}{|c|c|c|c|c|c|c|c|c|c|c|c|c|c|c|c|c|c|c|c|}
\hline \multirow{2}{*}{ Application } & \multirow{2}{*}{ No. } & \multicolumn{6}{|c|}{ Class I } & \multicolumn{5}{|c|}{ Class II } & \multicolumn{2}{|c|}{ Class III } & \multirow{2}{*}{$\begin{array}{c}\text { Class IV } \\
007-5 \\
\text { MS } \\
\end{array}$} & \multirow{2}{*}{$\begin{array}{c}\text { Class V } \\
\text { HT5 }\end{array}$} & \multicolumn{2}{|c|}{ CV (\%) } & \multirow{2}{*}{ Max/Min } \\
\hline & & $\begin{array}{l}\text { HP-5 } \\
\text { MS }\end{array}$ & DB-5 & ZB-5 & $\begin{array}{l}\text { DB-5 } \\
\text { HT }\end{array}$ & $\begin{array}{c}\text { ZB-5 } \\
\text { HT }\end{array}$ & $\begin{array}{l}\text { Rxi-5 } \\
\text { HT }\end{array}$ & $\begin{array}{l}\text { DB-5 } \\
\text { MS }\end{array}$ & Rxi-5 & $\begin{array}{l}\text { Inert } \\
\text { Cap }\end{array}$ & CP-Sil & $\begin{array}{l}\text { VF-5 } \\
\mathrm{ms}\end{array}$ & $\begin{array}{l}\text { TR-5 } \\
\text { MS }\end{array}$ & BPX-5 & & & $\begin{array}{c}\text { Inert Cap } \\
3 \text { lots }\end{array}$ & $\begin{array}{c}\text { All } \\
\text { column }\end{array}$ & \\
\hline \multirow[t]{2}{*}{ Is } & B47 & $2.9 \times 10^{6}$ & & $2.4 \times 10^{6}$ & & $3.1 \times 10^{6}$ & & $2.7 \times 10^{6}$ & & $3.4 \times 10^{6}$ & & $5.4 \times 10^{6}$ & & $4.3 \times 10^{6}$ & & $2.8 \times 10^{6}$ & & & \\
\hline & & & $3.8 \times 10^{6}$ & & $3.9 \times 10^{6}$ & & $3.0 \times 10^{6}$ & & $2.3 \times 10^{6}$ & & $4.3 \times 10^{6}$ & & $3.2 \times 10^{6}$ & & $2.1 \times 10^{6}$ & & & & \\
\hline \multirow[t]{17}{*}{ Lubricant } & B1 & 0.68 & 0.73 & 0.82 & 0.77 & 0.78 & 0.87 & 0.73 & 0.76 & 0.69 & 0.70 & 0.65 & 0.71 & 0.73 & 0.89 & 0.99 & 4 & 12 & 1.5 \\
\hline & B3 & 0.63 & 0.66 & 0.72 & 0.71 & 0.71 & 0.75 & 0.65 & 0.67 & 0.61 & 0.60 & 0.56 & 0.61 & 0.67 & 0.82 & 0.91 & 4 & 13 & 1.6 \\
\hline & B4 & 0.25 & 0.25 & 0.24 & 0.37 & 0.40 & 0.34 & 0.31 & 0.24 & 0.22 & 0.22 & 0.21 & 0.25 & 0.28 & 0.32 & 0.40 & 12 & 23 & 1.9 \\
\hline & B5 & 0.62 & 0.67 & 0.70 & 0.80 & 0.85 & 0.77 & 0.77 & 0.61 & 0.56 & 0.55 & 0.52 & 0.62 & 0.71 & 0.80 & 1.03 & 10 & 19 & 2.0 \\
\hline & B6 & 0.48 & 0.47 & 0.46 & 0.47 & 0.48 & 0.48 & 0.46 & 0.50 & 0.48 & 0.47 & 0.45 & 0.48 & 0.45 & 0.55 & 0.48 & 4 & 5 & 1.2 \\
\hline & B8 & 0.22 & 0.23 & 0.29 & 0.24 & 0.29 & 0.23 & 0.20 & 0.21 & 0.19 & 0.20 & 0.16 & 0.18 & 0.19 & 0.22 & 0.27 & 2 & 17 & 1.8 \\
\hline & B9 & 0.51 & 0.50 & 0.48 & 0.52 & 0.55 & 0.52 & 0.50 & 0.53 & 0.50 & 0.48 & 0.47 & 0.53 & 0.48 & 0.56 & 0.50 & 3 & 5 & 1.2 \\
\hline & B10 & 0.13 & 0.13 & 0.18 & 0.15 & 0.16 & 0.16 & 0.14 & 0.15 & 0.16 & 0.15 & 0.13 & 0.13 & 0.14 & ND & 0.11 & 17 & 13 & - \\
\hline & B11 & 0.12 & 0.11 & 0.15 & 0.13 & 0.14 & 0.14 & 0.12 & 0.11 & 0.13 & 0.13 & 0.11 & 0.10 & 0.11 & ND & 0.08 & 12 & 15 & - \\
\hline & B12 & 0.11 & 0.10 & 0.13 & 0.14 & 0.15 & 0.14 & 0.12 & 0.10 & 0.11 & 0.11 & 0.11 & 0.09 & 0.10 & ND & 0.08 & 10 & 18 & - \\
\hline & B14 & 0.34 & 0.36 & 0.42 & 0.34 & 0.32 & 0.35 & 0.36 & 0.41 & 0.38 & 0.37 & 0.37 & 0.40 & 0.36 & 0.42 & 0.35 & 7 & 8 & 1.3 \\
\hline & B15 & 0.36 & 0.35 & 0.39 & 0.33 & 0.33 & 0.36 & 0.38 & 0.40 & 0.38 & 0.38 & 0.34 & 0.38 & 0.35 & 0.44 & 0.32 & 8 & 9 & 1.4 \\
\hline & B16 & 0.39 & 0.36 & 0.40 & 0.37 & 0.37 & 0.36 & 0.39 & 0.41 & 0.38 & 0.38 & 0.35 & 0.38 & 0.36 & 0.46 & 0.34 & 8 & 7 & 1.4 \\
\hline & B17 & 0.42 & 0.42 & 0.41 & 0.42 & 0.44 & 0.37 & 0.40 & 0.40 & 0.36 & 0.41 & 0.38 & 0.42 & 0.40 & 0.45 & 0.37 & 13 & 6 & 1.3 \\
\hline & B18 & 0.43 & 0.35 & 0.42 & 0.44 & 0.48 & 0.39 & 0.43 & 0.38 & 0.36 & 0.40 & 0.38 & 0.47 & 0.44 & 0.43 & 0.35 & 5 & 10 & 1.4 \\
\hline & B20 & 0.35 & 0.32 & 0.32 & 0.40 & 0.43 & 0.39 & 0.38 & 0.32 & 0.34 & 0.31 & 0.29 & 0.33 & 0.34 & 0.38 & 0.38 & 6 & 11 & 1.5 \\
\hline & B21 & 0.45 & 0.50 & 0.54 & 0.46 & 0.49 & 0.48 & 0.47 & 0.54 & 0.53 & 0.50 & 0.51 & 0.58 & 0.48 & 0.55 & 0.13 & 3 & 12 & 4.5 \\
\hline \multirow{12}{*}{ Antioxidant } & B22 & 0.73 & 0.80 & 0.95 & 0.83 & 0.88 & 0.81 & 0.75 & 0.88 & 0.89 & 0.81 & 0.83 & 1.02 & 0.77 & 0.97 & 0.54 & 16 & 40 & 1.9 \\
\hline & B23 & 0.72 & 0.77 & 0.93 & 0.89 & 0.94 & 0.81 & 0.76 & 0.86 & 0.87 & 0.78 & 0.78 & 1.01 & 0.77 & 0.98 & 0.53 & 10 & 22 & 1.9 \\
\hline & B24 & 0.48 & 0.50 & 0.58 & 0.62 & 0.67 & 0.53 & 0.54 & 0.50 & 0.54 & 0.47 & 0.51 & 0.62 & 0.49 & 0.45 & ND & 12 & 14 & - \\
\hline & B25 & 0.36 & 0.34 & 0.33 & 0.40 & 0.41 & 0.37 & 0.40 & 0.35 & 0.33 & 0.34 & 0.30 & 0.35 & 0.37 & 0.41 & $<0.1$ & 12 & 15 & $>4$ \\
\hline & B26 & 0.61 & 0.59 & 0.65 & 0.85 & 0.94 & 0.74 & 0.62 & 0.60 & 0.64 & 0.58 & 0.56 & 0.68 & 0.58 & 0.74 & 0.20 & 7 & 12 & 4.7 \\
\hline & B27 & 0.29 & 0.27 & 0.29 & 0.38 & 0.40 & 0.35 & 0.29 & 0.28 & 0.30 & 0.27 & 0.27 & 0.34 & 0.27 & 0.30 & $<0.1$ & 8 & 25 & $>4$ \\
\hline & B28 & 0.19 & 0.20 & 0.18 & 0.23 & 0.25 & 0.21 & 0.21 & 0.19 & 0.20 & 0.18 & 0.16 & 0.22 & 0.21 & 0.21 & 0.12 & 12 & 25 & 2.1 \\
\hline & B31 & 0.30 & 0.31 & 0.30 & 0.42 & 0.44 & 0.30 & 0.41 & 0.24 & 0.31 & 0.23 & 0.27 & 0.38 & 0.34 & 0.27 & ND & 4 & 16 & - \\
\hline & B36 & 0.28 & 0.29 & 0.26 & 0.34 & 0.35 & 0.28 & 0.30 & 0.25 & 0.28 & 0.24 & 0.23 & 0.31 & 0.30 & 0.27 & ND & 4 & 21 & - \\
\hline & B37 & 0.16 & 0.19 & 0.20 & 0.28 & 0.29 & 0.24 & 0.18 & 0.10 & 0.10 & 0.10 & $<0.1$ & 0.10 & 0.14 & 0.14 & 0.22 & 5 & 22 & $>2$ \\
\hline & B40 & 0.22 & 0.22 & 0.21 & 0.30 & 0.35 & 0.23 & 0.29 & 0.18 & 0.23 & 0.19 & 0.16 & 0.27 & 0.24 & 0.24 & 0.18 & 11 & 24 & 2.2 \\
\hline & B41 & 0.36 & 0.36 & 0.42 & 0.45 & 0.47 & 0.39 & 0.36 & 0.40 & 0.39 & 0.36 & 0.36 & 0.46 & 0.37 & 0.43 & ND & 4 & 10 & - \\
\hline \multirow[t]{6}{*}{$\begin{array}{l}\text { Ultraviolet } \\
\text { absorber }\end{array}$} & B42 & 0.12 & 0.13 & 0.13 & 0.14 & 0.16 & 0.12 & 0.12 & 0.13 & 0.12 & 0.12 & 0.11 & 0.14 & 0.13 & $<0.1$ & $<0.1$ & 4 & 21 & - \\
\hline & B43 & 0.37 & 0.35 & 0.38 & 0.59 & 0.63 & 0.43 & 0.40 & 0.29 & 0.34 & 0.31 & 0.35 & 0.44 & 0.37 & 0.15 & ND & 2 & 24 & - \\
\hline & B44 & 0.41 & 0.41 & 0.45 & 0.52 & 0.54 & 0.46 & 0.43 & 0.43 & 0.42 & 0.40 & 0.37 & 0.50 & 0.43 & 0.51 & 0.16 & 4 & 15 & 3.4 \\
\hline & B45 & 0.23 & 0.23 & 0.23 & 0.29 & 0.32 & 0.25 & 0.24 & 0.22 & 0.24 & 0.21 & 0.19 & 0.26 & 0.24 & 0.26 & 0.16 & 4 & 15 & 2.0 \\
\hline & B46 & 0.57 & 0.55 & 0.53 & 0.69 & 0.74 & 0.60 & 0.59 & 0.53 & 0.58 & 0.51 & 0.46 & 0.62 & 0.58 & 0.61 & 0.38 & 4 & 30 & 1.9 \\
\hline & B48 & 1.53 & 1.46 & 1.51 & 2.01 & 2.23 & 1.76 & 1.57 & 1.42 & 1.51 & 1.36 & 1.32 & 1.81 & 1.50 & 1.77 & 1.22 & 4 & 17 & 1.8 \\
\hline
\end{tabular}

B47 was used as an internal standard (Is), the values show peak areas. Each value is the average of three analytical runs. ND: not detected. 
ticizers, and two benzophenone type ultraviolet absorbers could not be detected using some columns. Judicious selection of the column used for analysis of these additives is thus recommended.

\section{REFERENCES}

[1] M. Peltzer, J. Wagner and A. Jime'nez, "Migration Study of Carvacrol as a Natural Antioxidant in High-Density Polyethylene for Active Packaging," Food Additives and Contaminants, Vol. 26, No. 6, 2009, pp. 938-946.

[2] J. H. Petersen and L. K. Jensen, "Phthalates and FoodContact Materials: Enforcing the 2008 European Union Plastics Legislation," Food Additives and Contaminants, Vol. 27, No. 11, 2010, pp. 1608-1616. doi:10.1080/19440049.2010.501825

[3] A. Guart, F. Bono-Blay, A. Borrell and S. Lacorte, "Migration of Plasticizers, Phthalates, Bisphenol A, and Alkylphenols from Plastic Containers and Evaluation of Risk," Food Additives and Contaminants, Vol. 28, No. 5, 2011, pp. 676-685. doi:10.1080/19440049.2011.555845

[4] C. Simoneau, L. V. Eede and S. Valzacchi, "Identification and Quantification of the Migration of Chemicals from Plastic Baby Bottles Used as Substitutes for Polycarbonate," Food Additives and Contaminants, Vol. 29, No. 3, 2012, pp. 469-480.

[5] L. L. Barnkob and J. H. Petersen, "Effect of Relative Humidity on the Migration of Benzophenone from Paperboard into the Food Simulant Tenax and Modeling Hereof," Food Additives and Contaminants Part A, Vol. 30, No. 2, 2013, pp. 395-402. doi:10.1080/19440049.2012.741717

[6] Y. Kawamura, K. Watanabe, K. Sayama, Y. Takeda and T. Yamada, "Simultaneous Determination of Polymer Additives in Polyethylene by GC/MS," Journal of the Food Hygienic Society of Japan, Vol. 38, No. 5, 1999, pp. 307-318. doi:10.3358/shokueishi.38.5 307

[7] Y. Kawamura, C. Tagai, T. Maehara and T. Yamada, "Additives in Polyvinyl Chloride and Polyvinylidene Chloride Products," Journal of the Food Hygienic Society of Japan, Vol. 40, No. 4, 1999, pp. 274-284. doi:10.3358/shokueishi.40.4 274

[8] Y. Kawamura, C. Tagai, T. Maehara and T. Yamada, "Simultaneous Determination Method of Additives in Polyvinyl Chloride," Journal of the Food Hygienic Society of Japan, Vol. 40, No. 3, 1999, pp. 189-197. doi:10.3358/shokueishi.40.3 189

[9] Y. Kawamura, R. Yonezawa, T. Maehara and T. Yamada, "Determination of Additives in Food Contact Polypropylene," Journal of the Food Hygienic Society of Japan, Vol. 40, No. 2, 2000, pp. 154-161. doi:10.3358/shokueishi.41.154

[10] Y. Abe, M. Yamaguchi, M. Mutsuga, Y. Hirahara and Y. Kawamura, "Survey of Plasticizers in Polyvinyl Chloride Toys," Journal of the Food Hygienic Society of Japan, Vol. 53, No. 1, 2012, pp. 19-27. doi:10.3358/shokueishi.53.19

[11] European Commission, "Commission Regulation 10/2011/ EU of 14 January 2011 on Plastic Materials and Articles Intended to Come into Contact with Food," Official Journal of the European Union, 2011, pp. 1-89. 